\title{
Management and therapeutic response of a prostate ductal adenocarcinoma: a still unknown tumour?
}

 \\ ${ }^{1}$ Department of Urology, University of Modena, Modena - Italy \\ ${ }^{2}$ Department of Pathology, University of Modena, Modena - Italy \\ ${ }^{3}$ Department of Oncology, University of Modena, Modena - Italy \\ ${ }^{4}$ Department of Urology, Tanta University, Tanta - Egypt
}

\begin{abstract}
Clinical practice points
Ductal adenocarcinoma is a rare subtype of prostate cancer with a worse prognosis.

Histologically, it is characterized by the presence of tall, pseudostratified columnar epithelium with abundant cytoplasm organized in a papillary or cribriform-papillary pattern. Several clinical differences distinguish this subtype of prostate cancer by the conventional acinar adenocarcinoma: exophytic growth into the prostatic urethra, different clinical presentation, different sites of metastasis and more aggressiveness. The rarity of this tumour forced to base our knowledge on small case series or on individual case reports, and does not help to establish appropriate guidelines. Therefore, the diagnosis of this tumour masks clinical implications that are still not well-understood. We report the case of a 69-year-old Caucasian man with a diagnosis of pure prostate ductal adenocarcinoma that early developed multiple metastases after radical prostatectomy. The patient started hormonal therapy with a fast biochemical and radiologic (positron emission tomography-computed tomography, PET-CT) hormonal escape. Therefore, we took the decision to perform chemotherapy with Taxotere along with prednisolone with a relative stability of prostate-specific antigen (PSA) level, but a new PET-CT scan showed a further progression of the disease. Finally, the patient underwent therapy with Abiraterone acetate that did not stop the cancer progression. No therapeutic options available showed a good control of disease progression. PSA proved to be a poor marker while, on the contrary, PET-CT scan has proved to be particularly useful in the management of the disease progression. More efforts are required to add new knowledge about this tumour and assess what is known until now.
\end{abstract}

Keywords: Clinical management, Follow-up, Prostate, Prostate ductal adenocarcinoma, Therapeutic response

\section{Introduction}

Ductal adenocarcinoma of the prostate (PDA) is a rare histological subtype, accounting for only $1-5 \%$ of all prostate cancers (PCa). It was initially described by Melicow and Pacher in 1967 (1).

Histologically, it is characterized by the presence of tall, pseudostratified columnar epithelium with abundant cytoplasm organized in a papillary or cribriform-papillary pattern. It can occur as a pure form or, more often, mixed with the typical adenocarcinoma pattern. With respect to histology, it has two growth patterns: type $A$, an exuberant papillary endometrioid pattern with a focal intraductal component, and

\section{Accepted: March 29, 2016}

Published online:

\section{Corresponding author:}

Giacomo Maria Pirola, MD

Policlinico di Modena, Department of Urology

Via del Pozzo, 71 Modena, 41124, Italy

gmo.pirola@gmail.com type B, less papillary-endometrioid growth and more intraductal components. The specimen of PDA results positive for prostate-specific antigen (PSA) and focally for mucin, confirming the prostatic epithelial origin of this tumour. PDA is detected in both the periurethral region and peripheral zone of the prostate and is considered high grade in the modified Gleason grading system. It is an aggressive tumour with a shortened average time to progression and worse prognosis compared with acinar adenocarcinoma (AA) (2).

Its clinical behaviour shows a number of differences in comparison with the well-known AA. Tumours displayed exophytic growth into the prostatic urethra, with involvement of the verumontanum. The urethral orifices of the primary prostatic ducts can be uniformly involved. Obstructive symptoms or haematuria may develop at an early stage when the tumour is still small, and rectal examination is often normal or only suggestive of benign prostatic disease (3). The propensity of this tumour to spread within the urothelium makes local failure of common curative therapy (4).

Sometimes patients may present with secondary infertility and progressive decrease of ejaculatory volume and unusual metastases (testicle, penis and skin) were described in 


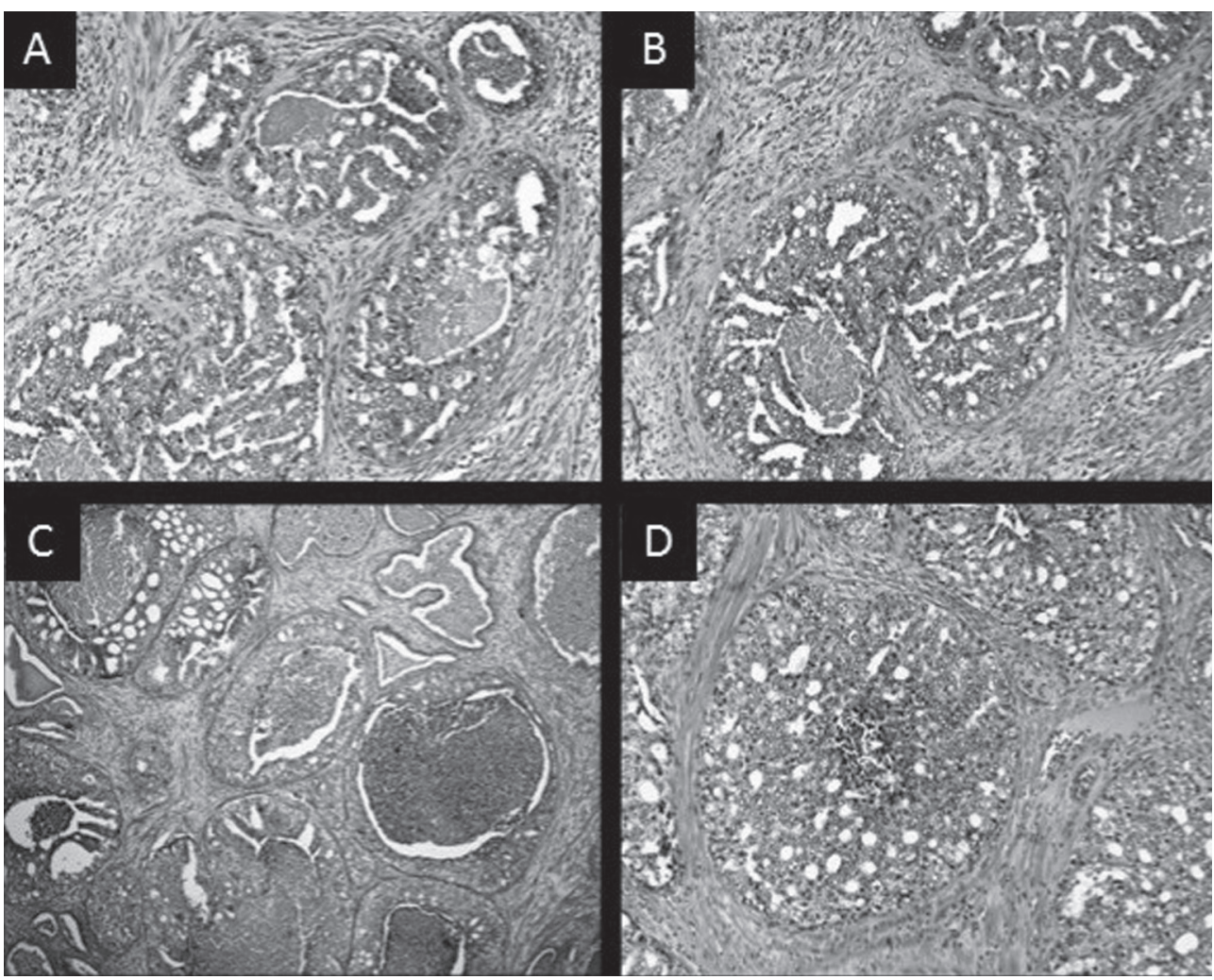

[AU: Ple Fig. 1 - Prost actr ductal adenocarcinoma type (B) All figures are in ematoxylin and eosin stain (A-B-D) is in 20x High-power field; (C) is at 10x High-power field). (A, B) and (D): The tumour shows the typical complex cribriform growth. (C): Comedonecorsis.

patients with this subtype of tumour $(5,6)$. Finally, the rarity of this tumour forced to base our knowledge on small case series or on individual case reports and do not help to establish appropriate guidelines. In fact, some aspects of this cancer are still unclear and insufficient data are available regarding its response to conventional therapies especially after biochemical recurrence. Therefore, its management does not differ from that of the well-known AA, although the diagnosis of this tumour masks clinical implications that are not still well understood.

This report presents a description of clinical management and therapy response of a pure PDA case in a patient with malignancy recurrence after radical prostatectomy.

\section{Discussion}

A 69-year-old Caucasian man presented in our Department in February 2011 with an elevated PSA value $(6.3 \mathrm{ng} / \mathrm{ml})$ and obstructive urinary symptoms in the form of increased urgency and nocturia. Informed consent for scientific divulgation of data was obtained before all procedures. Digital rectal examination (DRE) was negative for prostate nodules. He did a 20 cores transrectal ultrasound-guided (TRUS) biopsy. This examination revealed a Gleason Score $8(4+4)$ PDA in 10/20 cores. The remaining $10 / 20$ cores were cancer free. Initial bone scan and abdominal computed tomography (CT) were negative for metastatic disease. The radical prostatectomy and bilateral pelvic lymph node dissection (totally 11 L.Ns for right side and 8 L.Ns for left side) was performed.

Histopathology examination confirmed the presence of a pure Gleason score $8(4+4)$ prostate confined PDA without nodal metastasis (pT2c NO Mx Gleason 8 R0) (Fig. 1). Two months later, PSA value was $0.01 \mathrm{ng} / \mathrm{ml}$, but it increases in the checks carried out 3, 6 and 8 months later $(0.02,0.29$ and $1.36 \mathrm{ng} / \mathrm{ml}$, respectively) (Fig. 2). The patient underwent PETCT 11-choline to identify local or distant recurrences of the disease. The PET- CT scan identified two pulmonary nodules (approximately $1 \mathrm{~cm}$ ) with a tracer uptake (apical segment of right lower lung lobe and anterior segment of left apical lung lobe) (Fig. 3A).

Therefore, we took the decision to perform CT-guided biopsy of the lung nodules to assess a histological confirmation of distant recurrent PDA and to exclude a primitive lung tumour (alveolar bronchiolar carcinomas can pick up choline). This examination confirmed the presence of metastatic $\mathrm{PCa}$ positive for PSA. Thus, the patient started hormonal therapy with LH-RH analogue (triptorelin pamoate 11.25) converted in major androgenic bloc (MAB) 6 months later (leuprolelin acetate $22.5 \mathrm{mg}$ and bicalutamide $50 \mathrm{mg}$ ) for the partial response of disease (PSA decreased to $0.02 \mathrm{ng} / \mathrm{ml}$, but after 3 months, it starts to arise again becoming $0.3 \mathrm{ng} / \mathrm{ml}$ in the following 6 months). Eight months later, no control of disease was obtained (PSA was still raising up $2 \mathrm{ng} / \mathrm{ml}$ ) (Fig. 2). We took the decision to repeat a PET-CT scan (Fig. 3B) that showed an incongruity between the PSA level and the important progression of disease (significant increase of all known lesions, onset of a new VIII left rib metastasis and development of a new nodal mass with intense uptake of choline in left lung hilum). These findings preceded the slowly subsequent increase of PSA value in the following 10 months. Therefore, when PSA became $7.34 \mathrm{ng} / \mathrm{ml}$, we took the decision to perform six cycles of chemotherapy with 3-weekly 


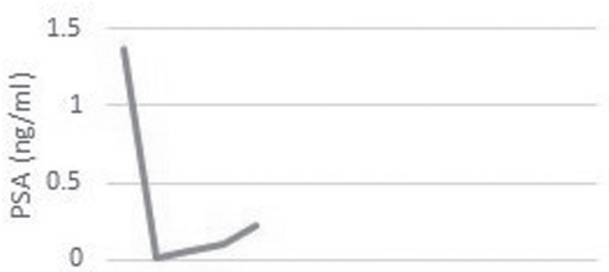

03689

Months

Triptor elina treatment response

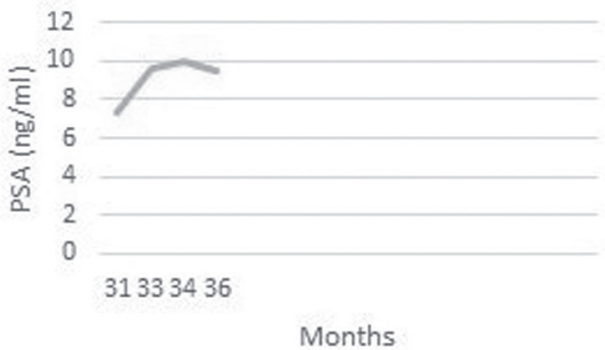

Taxotere response

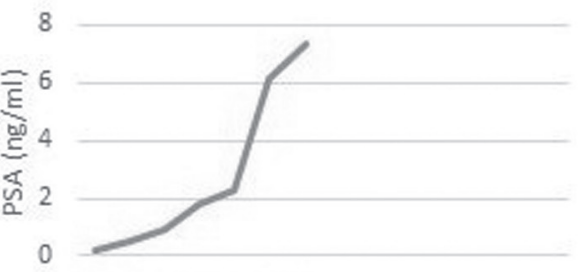

9151720242931

Months

MAB response

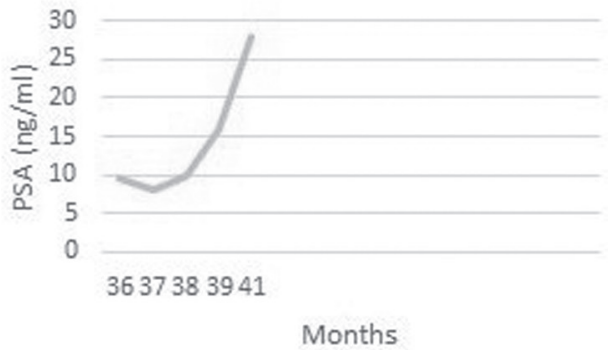

- Abirateronetreatment response
Fig. 2 - PSA value in relation to different treatment lines.


Taxotere (Docetaxel ${ }^{\circledR}$ ) $75 \mathrm{mg} / \mathrm{m}^{2}$ along with prednisolone $5 \mathrm{mg}$ twice-daily scheme. Close follow-up demonstrated a relative stability of the disease during chemotherapy based on PSA level (Fig. 2), but a new PET-CT was performed with evidence of a further progression of all known previous lesions aggravated by development of multiple (at least 10 new lesions) bilateral lung metastases (Fig. 3C). According with
Fig. 3 - PET-CT progression of disease. (A) (after radical prostatectomy): Metastases involve apical segment of right lower lung lobe and anterior segment of left apical lung lobe. (B) (after hormonal therapy): Onset of a new VIII left rib metastases and development of a new nodal mass with intense capitation of choline in left lung hilum. (C) (after chemotherapy): Progression of all know previous findings aggravated by development of multiple bilateral lung metastases. these findings, the decision was to switch chemotherapy to VII cycles of Abiraterone acetate (Zytiga ${ }^{\circledR}$ ) 1000 mg daily administration, but no significant response was observed and the PSA value increased until $27.98 \mathrm{ng} / \mathrm{ml}$ in the following 5 months (Fig. 2). One month later, PSA raised up to $150 \mathrm{ng} / \mathrm{ml}$. A new radiological valuation showed a further progression of metastatic disease and the patient died some weeks later. 
This case is emblematic of the variable and unpredictable behaviour of this rare histological type of PCa. The evidence of pulmonary metastases with an initial organ-confined prostate cancer after prostatectomy (negative resection margins without evidence of lymph node and bone metastases) may suggest a different natural course of PDA. A review of the literature revealed that visceral (lung and liver) metastases were relatively common in PDA compared with usual prostate AA (3). PDA may have the propensity to haematogenous spread rather than lymphatic in contrast to purely $A A$ variant. In our opinion, this should be related to the histopathological growth pattern and to the anatomic distribution of cancer within the gland. The development into glandular ducts, rather than inside the glandular berry, could facilitate direct vascular invasion instead of involvement of the glandular interstice or of the periacinar lymphatic vessels.

Even though PDA cell produces PSA, the serum concentration of this marker is not elevated in all patients. Morgan et al (7) stated that a number of patients with PDA should have PSA level less than $4.0 \mathrm{ng} / \mathrm{ml}$ at the diagnosis. Therefore, PDA is less likely detectable based only on PSA serum level. On the basis of these evidences, the common tools used for the diagnosis, staging and follow-up of PCa may not have the same validity for this tumour subtype. Some Authors suggest adopting other alternative markers such as carcinoembryonic antigen (CEA); however, the small number of reported cases limits the significance of this observation (5). Considering the above reasons, we think that a contemporary and steady radiological examination should be useful to manage this tumour. PET-CT scan plays, in this experience, a role of particular importance in the disclosure of metastasis progression and its therapeutic management. Although the PSA serum values were in steady slight increase, PET-CT scan showed a faster progression of the recurred disease (faster increase of volume and activity of mediastinal lesions and metastatic involvement on other sites). Thus, in our opinion, a radiological examination is necessary because it should help urologist to establish, with more precision, the appropriate timing for therapeutic decisions. Moreover, it is possible that the cancer had already given unrecognized lung metastases at primary tumour diagnosis. PET-CT scan (performed preoperatively) gives a reliable total body examination and could improve the diagnosis of metastases not revealed using routinely tests recommended for PCa staging (i.e. abdomen CT and bone scan).

Finally, the PDA response to hormone therapy is still debatable showing controversial statements. Millar et al. found an excellent and long-term response (mean 5.4 years) to the endocrine control based on PSA level (8). In our experience, hormone therapy does not give an adequate control of disease. The PSA trend during hormonal therapy, in our case, might suggest the presence of a primitive castrationresistant cancer or a fast hormonal escape conversion of the tumour (Fig. 2). Moreover, the PET-CT showed a rapid disease progression that was unexpected considering the PSA value alone.

In a case report, Aalok et al (9) underline, for the first time, as the chemotherapy (taxotere $75 \mathrm{mg}$ along with prednisone $5 \mathrm{mg}$ daily scheme), quickly adopted after biochemical recurrence, assured the control of a PDA metastatic disease for more than 27 months (reduction of imaging findings and of the PSA level). Unfortunately, no other data are available to compare these results. In our experience, the same chemotherapy scheme showed only a 5 months partial control of PSA level (ranged from 7.5 to $9.5 \mathrm{ng} / \mathrm{ml}$ ) not confirmed by PET-CT findings (progression of all known previous findings aggravated by development of multiple bilateral lung metastases).

Edgar Linden-Castro et al. (10), for the first time, observed that a patient treated with Abiraterone acetate $1000 \mathrm{mg}$ had a PSA progression time of 9 months and radiographic progression-free survival of 11 months. In our experience, Abiraterone acetate did not stop the progression of the PSA, which recorded a new peak of increase up to $27 \mathrm{ng} / \mathrm{ml}$. Unfortunately, a new radiological evidence is not still available, but we can expect a new important disease progression.

Summarizing, in our experience, the available therapies used for metastatic PCa treatment seem to slow down but not to stop the disease progression of PDA with a survival after recurrence of about 45 months (significantly less if compared with a GI 8 typical AA). Probably, some histological characteristics (pure or mixed, growth pattern Type A or Type B) or other still unknown factors may affect the tumour response to therapy and explain the differences between our findings and those of other cases. So, more efforts are required to add new knowledge about the biology of this tumour and assess what is known until now.

\section{Conclusion}

Our findings confirm that PDA is a more aggressive variant of PCa. PSA may be an insufficient marker for disease management and a partial or complete PSA response to therapy does not necessarily mean lack of disease progression. New markers or radiological evaluation should be applied to improve the management of this aggressive variant and, in our experience, PET -CT scan has proved to be particularly useful. We observed that the available cures seem to slow down but not stop the disease progression. Probably, some histological characteristics or other still unknown factors may affect the tumour response to therapy. More efforts are required to add new knowledge about the biology of this tumour and assess what is known until now.

\section{Disclosures}

Financial support: The authors have no financial disclosures to make. Conflict of interest: The authors have no conflict of interest.

\section{References}

1. Melicow M, Pachter M. Endometrial carcinoma of the prostatic utricle (uterus masculinus). Cancer. 1967;20:1715-1721

2. Samaratunga $H$, Delahunt B. Ductal adenocarcinoma of the prostate: current opinion and controversies. Anal Quant Cytol Histol. 2008;30(4):237-246.

3. Bostwick DG, Kindrachuk RW, Rouse RV. Prostatic adenocarcinoma with endometrioid features. Clinical, pathologic, and ultrastructural findings. Am J Surg Pathol. 1985;9(8):595-09

4. Perrapato SD, Shah PC, Huben RP, Gaeta JF. Locally recurrent endometrioid adenocarcinoma of the prostate after radical prostatectomy. J Urol. 1991;145(2):373-375. 
5. Tu SM, Reyes A, Maa A, et al. Prostate carcinoma with testicular or penile metastases. Clinical, pathologic, and immunohistochemical features. Cancer. 2002;94(10):2610-2617

6. Collina G, Reggiani C, Carboni G. Ductal carcinoma of the prostate metastatic to the skin. Pathologica. 2011;103(2): 50-51.

7. Morgan TM, Welty CJ, Vakar-Lopez F, Lin WD, Wright JL. Ductal adenocarcinoma of prostate: increased mortality risk and decreased serum prostate specific antigen. J Urol. 2010;184: 2303-2307
8. Millar EK, Sharma NK, Lessells AM. Ductal (endometrioid) adenocarcinoma of the prostate: a clinicopathological study of 16 cases. Histopathology. 1996;29:11-19.

9. Kumar A1. Mukherjee SD. Metastatic ductal carcinoma of the prostate: a rare variant responding to a common treatment. Can Urol Assoc J. 2010;4(2):E50-E54.

10. Linden-Castro E, Pelayo-Nieto M, Alias-Melgar A, EspinosaPerezgrovas D, RamirezGalindo I, Catalan-Quinto G. Abiraterone acetate and castration resistant ductal adenocarcinoma of the prostate. Case Rep Urol. 2014; 2014:508305. 